\title{
1 Genotype Imputation and Reference Panel: A Systematic Evaluation
}

2

3 Wei-Yang Bai ${ }^{1,2}$, Xiao-Wei Zhu ${ }^{1,2}$, Pei-Kuan Cong ${ }^{1,2}$, Xue-Jun Zhang ${ }^{3}$, J Brent Richards ${ }^{4}$, Hou-

$4 \quad$ Feng Zheng ${ }^{1,2^{*}}$

51 Diseases \& Population (DaP) Geninfo Lab, School of Life Sciences, Westlake University, 18

6 Shilongshan Road, Hangzhou 310024, China.

72 Institute of Basic Medical Sciences, Westlake Institute for Advanced Study, 18 Shilongshan

8 Road, Hangzhou 310024, China.

93 Institute of Dermatology and Department of Dermatology, Huashan Hospital, Fudan University,

10 Shanghai, China.

114 Lady Davis Institute, Jewish General Hospital, McGill University, Montréal, Québec, Canada.

$12 *$ Corresponding to: Hou-Feng Zheng (zhenghoufeng@westlake.edu.cn)

14 Biographical note: Hou-Feng Zheng completed his M.D. and Ph.D. in China, and got trained as 15 postdoc in human genetics at McGill University, Canada. He is a member of American Society of 16 Human Genetics. 


\section{Abstract:}

18 Here, 622 imputations were conducted with 394 customized reference panels for Han Chinese and European populations. Besides validating the fact that the imputation accuracy could always benefit from the increased panel size when the reference panel was population-specific, the

21 results brought two new thoughts as follows. First, when the haplotype size of reference panel

22 was fixed, the imputation accuracy of common and low-frequency variants (MAF $>0.5 \%)$

23 decreased while the population-diversity of reference panel increased, but for rare variants

24 (MAF<0.5\%), a fraction of diversity $(<20 \%)$ of panel could improve the imputation accuracy.

25 Second, when the haplotype size of reference panel was increased with extra population-diverse

26 samples, the imputation accuracy of common variants (MAF>5\%) for European population could

27 always benefit from the expanding sample size. But for Han Chinese population, the accuracy of

28 all imputed variants reached the highest when reference panel contained a fraction of extra

29 diverse sample $(15 \% \sim 21 \%)$. In addition, we evaluated the existing reference panels such as the

30 HRC and 1000G Phase3 and CONVERGE. For European population, HRC was the best

31 reference panel. For Han Chinese population, we proposed an optimum constituent ratio for the

32 Han Chinese imputation if researchers would like to customize their own sequenced reference

33 panel, but a high quality and large-scale Chinese reference panel was still needed. Our findings

34 could be generalized to the other populations with conservative genome, a tool was provided to

35 investigate other populations of interest (https://github.com/Abyss-bai/reference-panel-

36 reconstruction). 
38 Key words: Genotype imputation; Reference panel; HRC; Chinese population.

\section{$40 \quad$ Highlights (Key points)}

41 1) A total of 394 reference panels were designed and customized by three strategies, and large-

42 scale genotype imputations were performed with these panels for systematic evaluation in Han

43 Chinese and European populations.

44 2) The accuracy of imputed variants reached the highest when reference panel contains a fraction

45 of extra diverse sample $(15 \%$ 21\%) for Han Chinese population, if the haplotype size of the

46 reference panel was increased with extra samples, which is the most common cases.

47 3) The imputation accuracy showed the different trends between Han Chinese and European

48 populations. In a sense, the European genome may more diverse than Han Chinese genome by

49 itself.

50 4) Existing reference panels were not the best choice for Chinese imputation, a high quality and

51 large-scale Chinese reference panel was still needed. 


\section{Introduction}

54 As a cost-efficient way of genotyping variants, imputation has become a standard approach in

55 genome-wide association studies (GWAS) in the past decade. It is achieved by using known

56 haplotypes in a population to infer initially-untyped genetic variants for testing association with a

57 trait of interest[1], thereby allowing to overcome one major limitation of SNP genotyping arrays.

58 In generally, SNP array only contains a small fraction of human genetic variation $\left(10^{5}-10^{6}\right)$,

59 genotype imputation makes these low-density genetic variants array become a higher one $\left(10^{7}-\right.$

$6010^{8}$ ). A higher-resolution view of a genetic region can provide many advantages for population

61 genetics research, such as guiding fine-mapping by increasing the chances of identifying a causal

62 variant[2], facilitating the combination of results across studies in meta-analysis[3, 4], and

63 increasing the power to detect an association signal[5, 6]. Since genotype imputation carries such

64 potentials, accuracy of imputed-variant is crucial. Many studies have illustrated the accuracy and reliability of genotype imputation in common variants (MAF > 5\%)[7-9]. Compared to common

66 variants, rare variants are often population specific and tend to have low levels of pairwise

67 linkage disequilibrium with other sites, but more likely to be associated with dramatic functional consequences[10, 11]. More and more rare and low-frequency variants were discovered to be associated with serious diseases[3, 12-14]. However, keep the imputation accuracy of rare and low-frequency variants at a reliable level is still a challenge[15].

71 Several imputation tools have been developed during the last decade, most of them employ the

72 hidden Markov model (HMM) as their engine, such as IMPUTE, MaCH and Minimac series[16- 
74 them is to reduce the compute pressure of server, the assistance they can provide in improving

75 accuracy of imputation of rare variants is very limited. Imputation reference panel as the

76 haplotype patterns and information carrier for inference of untyped genetic variants, its

77 composition and size are far more crucial influential factors for imputation accuracy[19].

78 Since the International HapMap 3 Project was accomplished in 2010[20], more and more whole-

79 genome sequencing (WGS) data were produced for public use. The quality of genotype imputation has benefited from the increase of genetic information in these publicly available

81 reference panels data[21, 22], one of the most famous and widely used reference panel is from

82 the 1000 Genomes Project (1000G)[23]. The 1000G Project Phase 3 identified more than 84.4 million single nucleotide polymorphisms (SNP) from 2,504 individuals which collected from 26

84 worldwide populations, each population contains 61 113 individuals. All of the 26 populations

85 were divided into 5 groups (AFR, African; AMR, Ad Mixed American; EAS, East Asian; EUR,

86 European; SAS, South Asian). Besides the 1000G Project, there are some more population-

87 specific reference panels. Examples include the UK10K Project[24] (3781 British sequenced at

$887 \times$ depth of coverage) and the Genome of the Netherlands[25] (GoNL, 250 Dutch parent-

89 offspring families sequenced at $14 \times$ depth). Recently, a large combined haplotype reference panel

90 named the Haplotype Reference Consortium (HRC) was formed, it consists of 64,976 haplotypes

91 at 39,235,157 SNPs with the minor allele count (MAC) greater or equal to 5, and it will collect

92 more WGS data in future[26]. In 2017, 11,670 genomes of Chinese sequencing project called

93 The China, Oxford and Virginia Commonwealth University Experimental Research on Genetic

94 Epidemiology (CONVERGE) was published, but only 22 million high quality SNPs were

95 identified because of its low-coverage sequencing depth (1.7×)[27]. 
96 There are many factors that affect imputation accuracy of rare variants, such as density of

97 genotyping array, ancestry diversity of GWAS data, as well as sequencing depth, haplotype size

98 and diversity of reference panel[1]. In general, for genetically diverse populations such as

99 Hispanics/Latinos in the USA, a corresponding diverse reference panel will improve the imputation accuracy[28]. For an ancestry-specific GWAS data, such as Southeast Asian[29] and

101 African ancestry[8], using the corresponding specific reference would gain more accuracy

102 because of the same genetic background. However, another study found that the accuracy of

103 imputation of low-frequency variants can benefit from the reference diversity, independent of

104 reference haplotype size[30]. It is widely accepted that the haplotype size is a key factor in a

105 particular reference panel, but mostly, expanding the reference panel size means to introduce

106 more population diversity.

107 Therefore, it is remaining unclear that the relationship between imputation accuracy of rare variants and composition of reference panels and how to maximize the imputation accuracy. Here, we proposed a much rigorous and systematic design to evaluate the relationships between imputation performance and haplotype diversity and size of reference panel for Han Chinese and

111 European populations, by using 394 customized reference panels and by performing 622

\section{Methods}

\section{Sample datasets and genotyping}


117 In this study, we used Han Chinese and European samples as GWAS sets. All Han Chinese

118 samples, which consist of 2,360 individuals, were obtained from multiple regions in central and

119 southern China[31]. The Illumina Human610-Quad (610K) BeadChip was employed for

120 genotyping analysis based on the Genome Reference Consortium Human build 36 (GRCh36) and

121 a total of 598,821 SNPs were identified. The European dataset was obtained from TwinsUK

122 Project (http://www.twinsuk.ac.uk/). All 3,461 European individuals were genotyped by using

123 the 610K BeadChip[32] which was the same as the Han Chinese genotyping array, and the

124 genome annotation was based on GRCh36.

\section{Quality control and pre-phasing}

126 We first updated the genome assembly version of genotyping array variants from build 36 to 37.

127 For all sample datasets, we performed a stringent quality control (QC) with four steps. Step one,

128 we retained autosomal bi-allelic SNPs with missing call rates $<=5 \%$ and samples with missing

129 call rates $<=2 \%$ of data by using PLINK v1.9[33]. Step two, the pairwise genetic relationship matrix between all samples was calculated by GCTA v1.91[34] using common variants with MAF $>10 \%$, and individuals with pairwise genetic relationship coefficient $>0.025$ will be thought to be cryptically related. We then randomly selected 2000 unrelated individuals for both

133 Han Chinese and European samples sets. Step three, we downloaded the legend file of $1000 \mathrm{G}$

Phase 3, and used the EAS (East Asians) and EUR (Europeans) populations as the reference to

135 check our Han Chinese and European data respectively by a Perl scripts of checking tools

136 (www.well.ox.ac.uk/ wrayner/tools/). We checked if any SNP ID or genome position was

137 mismatched with reference panel, if yes, the SNP was removed, and we corrected the allele

138 switch and strand flip in the GWAS sets, and we removed SNPs whose allele frequency 
139

140

141

142

difference with reference was larger than 0.2. Last step, we excluded the SNPs with missing call rates $>5 \%$ again, and excluded those deviating from Hardy-Weinberg equilibrium (HWE) at $\mathrm{P}<$ $1 \times 10^{-6}$. In order to study the imputation accuracy of very rare variants, we retained all SNPs in Chinese sample dataset. And note that SNPs in European were all with MAF > 0.5\%. Finally, 516,410 overlapped variants between Han Chinese and European in total were used as the study data.

To reduce the computed pressure of the subsequent large-scale genotype imputations, we phased Han Chinese and European datasets by using SHAPEIT v2.9[35] with the default settings in local server. And we checked our QCed data in Michigan imputation server[16].

\section{Reference panels re-construction by haplotype size}

Twenty-four out of the 26 populations in the $1000 \mathrm{G}$ Phase 3 reference panel with sample size greater than 85 were selected to study the relationship between haplotype size and imputation accuracy, that 3 were from AMR (Ad Mixed American), 6 were from AFR (African) and other 15 were from EAS (East Asian), SAS (South Asian) and EUR (European) respectively. We randomly and successively extracted $25,50,65$, and 85 samples from each population alone

(Figure 1a) and customized them into 4 size gradients, which were 50, 90, 130 and 170 haplotypes, and the higher gradient sets contained all haplotypes of the lower one. The bcftools was employed here (https://samtools.github.io/bcftools/). These customized 96 (24 times 4) panels were then used for imputation of Han Chinese and European sample sets by Minimac3 in local server, respectively (Figure 1a).

\section{Reference panels re-construction by population diversity}


160 We categorized 5 groups corresponded with EAS, EUR, AFR, AMR and SAS of populations

161 distribution in the 1000G Phase 3 to study the relationship between imputation accuracy and

162 diversity of reference panel (Figure 1b). Each group consists of 5 populations in sequence and

163 each population contains 64 samples (Supplementary Table 1). Note that AFR group has 7

164 populations in the $1000 \mathrm{G}$, we excluded the 'ASW' (Americans of African Ancestry in SW USA)

165 in AFR because of its small sample size, and re-categorized the 'ACB' (African Caribbeans in

166 Barbados) into AMR group in this study (see Discussion). The 5 groups of population that we

167 considered corresponded to a set of vectors $\left(i_{1}\right.$ to $\left.i_{5}\right)$, then we solved the function:

$i_{1}+i_{2}+i_{3}+i_{4}+i_{5}=5$

126 non-negative integer solutions were obtained in total. These solutions corresponded to 126

different combinations of 25 populations in 5 groups, the reference panels were then constructed

based on these combinations. For these panels, we set six levels of population diversity based on the number of populations of EAS or EUR, the diversity degree was raised from level to level ${ }_{5}$, for example, the solution $\left(i_{1}, i_{2}, i_{3}, i_{4}, i_{5}\right)=(2,3,0,0,0)$ means a reference panel consists of the first 2 populations in EAS, and the first 3 populations in EUR (Supplementary Table 1), and no AFR, AMR or SAS populations was included. Therefore, this panel was diverse for EAS at level 3 and for EUR at level 2 . Each panel contains 640 haplotypes. The imputations were performed for Han Chinese and European sample sets with 126 different diversity panels (Supplementary Table 2) by using Minimac3 in local.

\section{Reference panels re-construction by both haplotype size and population diversity}


180

181

182

183

184

185

186

187

188

189

190

191

192

193

194

195

196

197

In this part, a series of reference panels were customized with haplotype size and population

diversity constantly changed (Figure 1c). We took these two factors together as arguments to investigate the pattern of imputation accuracy variation. First, we extracted CHB (Chinese in Beijing) and CEU (Utah Residents with Northern and Western European Ancestry) population samples from 1000G Phase 3 according to the ancestry to our GWAS study sets. Besides, we also extracted other 10 populations included 3 AMR populations (PUR, CLM, PEL), 3 AFR

populations (YRI, LWK, GWD), 2 EUR populations (TSI, IBD) and 2 EAS populations (CHS,

JPT). The CHB and CEU population contains 103 and 99 samples respectively, and each of other 10 populations contains at least 85 samples. Then, we took CHB and CEU samples as two basic panels, and added other population samples to them constantly. To ensure that no individuals from corresponding specific group were involved in CHB-based and CEU-based panel, we used different adding strategies. For CHB-based panel, we chose the adding-populations from AMR, AFR and EUR groups. For CEU-based panel, we chose the adding-populations from AMR, AFR and EAS groups (Figure 1c), each group contained 3 populations, and then we respectively took one individual from these 9 populations per time, and recursively added them to basic panel for 85 times in total (Figure 1c). Finally, we got 172 imputation reference panels, half of them were CHB-based and another half were CEU-based. These panels were then used for Han Chinese and European sample sets imputation by using Minimac3 in local server, respectively.

\section{HRC, 1000G and CONVERGE reference panels}

The HRC was the largest reference panel for genotype imputation currently and mainly consist of European population samples[26]. The $1000 \mathrm{G}$ sample was consist of 26 worldwide populations and made it the most diverse reference panel[23]. The CONVERGE (The China, Oxford and 
202 Virginia Commonwealth University Experimental Research on Genetic Epidemiology) was a

203 Chinese-specific panel with 11,670 genomes with low depth $(1.7 \times)$ [27]. These three reference

204 panels were assessed in our study (Figure 1d). Minimac3 was employed for genotype imputation

205 in our study because of its advanced performance[16]. We converted all reference sets from

206 common VCF format into Minimac3 specialized M3VCF format which require lesser space and

207 are faster to read than VCF file while importing data.

208 A basic statistics of variants was performed first between HRC, 1000G and CONVERGE

209 reference panels. We used remote and local ways to impute our data, since the complete

210 haplotypes set of the HRC was not available for downloading yet, and the CONVERGE-based

211 imputation was performed using Minimac3 in local server. For consistency of imputation tools,

212 the Michigan Imputation Server was employed for remote HRC-based imputation. The 1000G-

213 based imputation was performed in both ways (Michigan Imputation Server and local imputation

214 server) to test the comparability of results of two different servers. Note that the $1000 \mathrm{G}$ panel had

215 two versions, one included singletons and another one not, the latter was mainly used here (see

216 Discussion). All imputations were conducted with default settings.

\section{Evaluation of imputation accuracy}

218 In this study, we employed Minimac3 statistics including $\mathrm{R}^{2}$ and empirical- $\mathrm{R}^{2}\left(\mathrm{EmpR}^{2}\right)$ to

219 evaluate genotype imputation quality and accuracy. Both $\mathrm{R}^{2}$ and $E m p \mathrm{R}^{2}$ value of each sites can

220 be obtained from imputation results. $\mathrm{R}^{2}$ was the estimated value of the squared correlation

221 between imputed genotypes and true, unobserved genotypes. Since true genotypes were not 
223 towards their expectations based on population allele frequencies alone[16]. $\mathrm{R}^{2}$ was defined as:

$$
\hat{r}^{2}=\frac{\frac{1}{2 n} \times \sum_{i=1}^{2 n}\left(D_{i}-\widehat{p}\right)^{2}}{\widehat{p}(1-\hat{p})}
$$

224 Where $p$ was the alternate allele frequency and $D_{i}$ was the imputed alternate allele probability at 225 the $i t h$ haplotype and $n$ was the number of GWAS study samples.

226 For each site that were genotyped in the study samples, Minimac3 can calculates a special

227 imputed dosage by hiding all known genotypes for that site. This imputed value is called leave-

228 one-out dosage (LooDosage) and was used to calculate EmpR ${ }^{2}$ by directly calculating the Pearson

229 correlation coefficient between LooDosage and known genotypes. Compared to $\mathrm{R}^{2}$, EmpR ${ }^{2}$ was

230 more powerful and effective to evaluate imputation accuracy, and can only be calculated in

231 genotyped sites. In our study, we set a strict threshold for 'well-imputed' sites, which the

232 Minimac3 $\mathrm{R}^{2}$ (imputation quality) had to reach at least 0.8 , and used Minimac3 $\mathrm{EmpR}^{2}$ to

233 measure the imputation accuracy.

\section{Results}

\section{Imputation accuracy versus haplotype size of panel}

237 We conducted a strict QC for Han Chinese and European genotyping array data sets in local, and checked the data after QC by using Michigan imputation server. The allele-frequency showed a strong correlation between GWAS sets and EAS or EUR data from the 1000G Phase 3 reference panels, which $\mathrm{r}^{2}=0.991$ for Han Chinese GWAS data set and $\mathrm{r}^{2}=0.994$ for European GWAS data 
241 set, respectively (Supplementary Figure 1). After QC, 516,410 overlapped sites in 2,000 unrelated

242 individuals retained respectively for both populations.

243 It is generally accepted that genotype imputation accuracy can benefit from increasing panel size.

244 In this study, we validated this point in a more systematic approach. We performed 192

245 imputations for Han Chinese and European GWAS data in this part. 24 worldwide populations

246 from 1000G Phase 3 were customized as reference panel, each population was transformed into 4

247 gradients according to the number of haplotypes. All different population panels showed the

248 consistent results that imputation accuracy increased with panel's haplotypes size for both

249 Chinese and European datasets (Figure 2a and 2b). For Han Chinese and European samples, the

250 average accuracy of all genotyped-variants reached the highest when we used CHB and CEU

251 population samples as the reference panel, respectively. To obtain the more distinct comparison

252 between gradientized reference panels, we divided variants into five MAF bins including: 5\% <=

$253 \mathrm{MAF}<100 \%, 1 \%<=\mathrm{MAF}<5 \%, 0.5 \%<=\mathrm{MAF}<1 \%, 0.1 \%<=\mathrm{MAF}<0.5 \%$ and $0.025 \%<$

254 MAF $<0.1 \%$. The imputation accuracy in different MAF bins all showed an increasing trend

255 when haplotypes size constantly augmented (Figure 2c and 2d). Besides, we counted well-

256 imputed $\left(\mathrm{R}^{2}>0.8\right)$ number of variants, the results showed that well-imputed variants number also

257 raised with haplotypes size (Supplementary Figure $2 \mathrm{a}$ and $2 \mathrm{~b}$ ). These results validated the fact

258 that the imputation quality could be improved by the haplotype size of reference panel.

259 The average EmpR ${ }^{2}$ results of comparing the Han Chinese imputation and European imputation

260 showed that the European population could be more accurately imputed when the corresponding

261 population reference panel was used (increasing from 0.82 to 0.87 for Han GWAS and CHB, and 

panels from EAS (Figure 3).

\section{Imputation accuracy versus population diversity of panel}

267 In this part, we constructed 126 "diversity" reference panels using the five population groups of the 1000G (Supplementary Table 2), and performed 252 imputations in total. The size of each reference panel was fixed to 640 haplotypes. The overall average EmpR ${ }^{2}$ decreased from 0.92 to accuracy.

281 We knew that the haplotypes size and populations diversity of reference panel were two crucial 
283

284

285

286

287

288

289

290

291

292

293

294

295

296

297

298

299

300

301

302

303

304

imputation accuracy of rare variants could benefits from the increasing of sample size and an appropriate proportion of diversity of reference panels, respectively. Most of the time, expanding sample size meant to introduce more diverse populations. In this part, we designed a series panel that the haplotypes size and populations diversity simultaneously raised for 85 times. A total of 172 panels were customized and divided into two groups (Figure 1c), sample size expanding from 103 to 868 for Han Chinese group (99 to 864 for European group) while population diversity augmented from 0 to $88 \%$. We found different patterns for Han Chinese and European imputation accuracy. For Han Chinese, the overall accuracy had an improvement at the beginning and reached the highest when panel's samples increased 3 step-size (27 individuals) with $21 \%$ population diversity introduced (Figure 5a). After that, the imputation accuracy continually decreased with diverse populations raised, but still higher than initial panel (0-step panel). However, for the European, the imputation accuracy showed a constantly increased trend from step one to the end with the sample size and diversity grew, the increases from the first step was most obviously (Figure 5a). This result suggested that the positive effect of sample-size-

expanding on imputation accuracy was not large enough to neutralize the negative effect of the panel diversity which introduced for Han Chinese after the diversity rare over $21 \%$. But it could offset the negative effect for European and improve the imputation accuracy, which meant that the imputation accuracy for European population could always benefit from the larger panel.

In the divided MAF bins of imputed-variants, the results showed the more detailed changes mode.

The imputation accuracy increased rapidly when the diverse population were introduced at the beginning for both populations, and then slowly decreased for the variants of Han Chinese population (Figure 5b) but slowly increased for the common variants of European population 
305 (Figure 5c) with diverse samples raised. Besides, for the variants with different MAF bins to

306 reach its highest accuracy, the diversity rate was increased when the variants got more and more

307 rare. These results suggested that an extra diversity of reference panel could remarkably improve

308 the imputation accuracy of rare variants, and appropriate proportion $(15 \% \sim 21 \%)$ diversity could

309 maximize it for Han Chinese population. Besides, we observed that the European population

310 could be more accurately imputed than the Han Chinese.

311 Based on the design of this part, we developed a panel re-construction tool for researchers to

312 investigate the imputation accuracy in other populations of $1000 \mathrm{G}$. User can set their own study

313 population, diverse populations, step size and adding times in an easily way, and customize a

314 series reference panel. This tool/package could be downloaded now from GitHub, and the details

315 of how to use this tool were included (https://github.com/Abyss-bai/reference-panel-

316 reconstruction).

\section{Imputation evaluation for the 1000G and HRC and CONVERGE panels}

318 Before actual imputation, the SNPs overlapping between three panels and distribution with seven

319 MAF bins were investigated (Supplementary Figure 3). The Venn diagram showed that 1000G P3,

320 HRC and CONVERGE has 15,524,045, 10,612,366 and 10,550,308 unique sites on autosomes

321 respectively, and all shared 10,303,072 sites in total (Supplementary Figure 4). The HRC-based

322 imputation was performed in Michigan server and the CONVERGE-based imputation was

323 performed in local server, the 1000G-based imputation was conducted in both ways (local and

324 remote), we compared the results of $1000 \mathrm{G}$-based imputation of two servers, the mean EmpR ${ }^{2}$

325 and imputed sites counts showed perfect consistency (Supplementary Figure 5 and 
326 Supplementary Table 3). This result illustrated that the bias caused by local and Michigan

327 imputation server's difference was negligible. Besides these three reference panels, in this part,

328 we also presented the "CHB21D" panel that consisted of CHB population and $21 \%$ extra diverse

329 samples (i.e. the 3-step panel for Han Chinese in last section) to compare its performance with the

330 three big reference panels.

331 For Han Chinese GWAS sets, the $1000 \mathrm{G}$ panel imputed 7,168,371 sites with $\mathrm{R}^{2}>=0.8$, which

332 was the best. The HRC panel showed the highest imputation quality with mean $\mathrm{R}^{2}=0.72$ in

333 shared sites and the CONVERGE panel showed the highest imputation accuracy with EmpR ${ }^{2}=$

3340.92 . However, due to only about 22 million sites in total were contained in the CONVERGE

335 panel, its number of well-imputed sites was the minimum $(5,626,185)$. For European GWAS sets,

336 the HRC panel resulted in 12,871,067 well-imputed $\left(\mathrm{R}^{2}>=0.8\right)$ sites which was the best among

337 the three panels and accounted for $32.9 \%$ of total imputed sites. And the HRC panel showed the

338 highest imputation quality with mean $\mathrm{R}^{2}=0.73$ in $10,302,818$ shared imputed sites, and showed

339 the highest imputation accuracy with a quite strong mean $\operatorname{EmpR}^{2}$ (0.98) (Table 1). We could

340 clearly know that HRC was the best panel for European samples genotype imputation from these

341 results, but for Chinese samples, however, each of three panels had their own advantages and

342 none of them was the most suitable panel.

343 We also divided imputed variants by MAF bins as above, but not focused on $>5 \%$ MAF bin. For

344 Han Chinese GWAS sets, the absolute number of well-imputed SNPs of the HRC and 1000G

345 panels was close and the most, the CONVERGE panel showed the minimum in four MAF bins,

346 and even lower than CHB21D panel (Figure 6a). The average $\mathrm{R}^{2}$ which represents imputation 
347 quality of variants showed that the CONVERGE panel was slightly better than 1000G panel

348 while the HRC was still the best (Supplementary Figure 6a). For European GWAS sets, the

349 absolute number of well-imputed variants and mean $\mathrm{R}^{2}$ of the HRC panel in all four MAF bins

350 were obviously higher than the corresponding values of the 1000G and CONVERGE panels

351 (Figure 6b and Supplementary Figure 6b). Moreover, even for the very rare variants whose MAF

352 in $0.025 \sim 0.1 \%$ bin, the HRC panel could well impute about 2.4 million sites while the $1000 \mathrm{G}$ and

353 CONVERGE panels could orefnly well impute 0.4 million and 2,021 sites respectively.

354 To obtain more comparable results, we extracted the 10,302,818 shared sites by three panels. The

355 HRC panel also showed the largest number of well-imputed variants and the highest average of

$356 \mathrm{R}^{2}$ in four MAF bins for both populations (Supplementary Figure 7). Besides, we found that, for

357 Han Chinese population, although the HRC imputed the most variants with $\mathrm{R}^{2}>0.8$, its EmpR $\mathrm{R}^{2}$

358 was just the lowest among four panels (Figure 7a). The Chinese-specific panel, CONVERGE, has

359 the highest imputation accuracy, and followed by the CHB21D panel which only contained 130

360 samples. The constitution of CHB21D panel showed the great potential at imputation accuracy.

361 For European imputation accuracy, the EmpR ${ }^{2}$ of the HRC panel was slightly higher than the

362 1000G panel, the CONVERGE panel was lowest (Figure 7b). These results implied that, for the

363 European population imputation, the HRC panel was the best choice, for the Chinese population,

364 a high quality and decent reference panel was still needed.

\section{Discussion}


367 In this study, by conducting 622 imputations with 394 customized reference panels for Han

368 Chinese and European populations, we found that when the haplotypes size of reference panel

369 was increased with extra population-diverse samples, which was usually the real case, the

370 imputation accuracy of Chinese population could reach the highest when reference panel contains

371 a fraction of extra diverse sample (15\% 21\%), but when the size of reference panel was fixed, the

372 pattern was different. In addition, we first evaluated the performance of Chinese-specific panel

373 CONVERGE and two frequently used reference panel HRC and 1000G. No doubt the HRC was

374 the best panel for European population since its performance on both imputation accuracy and

375 quality outperformed other panels. For Han Chinese population, the performance of the HRC and

376 1000G reference panel on well-imputed variants number were better than the CONVERGE panel,

377 but the CONVERGE showed the highest imputation accuracy. However, large-scale Han

378 population reference panel with high quality is remaining needed.

379 Since the first GWAS published in 2005, scientists showed a fantastic enthusiasm in this

380 powerful design to investigate the genetic risk factors for complex traits. A total of $\sim 3,700$

381 GWAS in complex trait/diseases have identified thousands of risk variants over the past 14

382 years[36], and GWAS will continue to contribute knowledge about population genetics in future.

383 The cost of one-human-genome sequencing has dropped to 1000 dollars in 2017[37] and even

384 lower by now, however, it is still too expensive to sequence samples in a large-scale study. With

385 the accomplishment and establishment of large sequencing projects and GWAS databases, such

386 as the 1000G project and UK biobank (UKB), more and more large-scale and high-depth

387 sequencing data went public and available. With reference panels that build from these high 
388

389

390

391

392

393

394

395

396

397

398

399

400

401

402

403

404

405

406

407

quality data, such as HRC, genotype imputation delivered an attractive low-cost alternative to sequencing.

Previous efforts have been focused on imputation evaluation for different populations, such as African, Chinese and Southeast Asian, by using publicly available reference panel[7, 8, 29], despite all these efforts, most studies have been conducted in a relatively shallow way because of the tremendous pressure to the computation server, and the main purpose of these studies were to evaluate the percentage of well imputed SNPs for a suitable reference panel. Less of them investigated how the factors affect the accuracy. Huang and Li's study designed a series of sizeunfixed reference panels using 210 HapMap samples which consist of four populations, and concluded that a mixed panel could lead to the maximal imputation accuracy for a particular population as its primary component was the same HapMap reference panel[19]. Their works investigated how to maximize the accuracy with HapMap reference panel, and raised the importance of size and composition of the reference panel, but was not detailed enough for a systemic study on imputation accuracy of rare variants. The genotype imputation required a high computing power, and the large-scale imputation study was mostly hindered by this requirement.

In this study, we randomly selected 2,000 unrelated individuals for the Han Chinese and the

European GWAS set, respectively. Actually, the GWAS set sample size would linearly increase the computed pressure. Usually, for a large-scale imputation accuracy study, people would prefer a smaller GWAS sample size, such as less than 1,000. Although the sample size we used would produce more computation load, it can do reduce the accuracy error and result a more precise and 
409 imputation (control the high missingness rate of samples and variants, high deviations from

410 Hardy-Weinberg equilibrium and high inbreeding coefficient etc), we checked our GWAS data

411 twice in case of the mistake that varied situations may bring in subsequent imputation, such as

412 SNP mismatch, allele switch and strand flip, by Michigan Imputation Server and local tools. We

413 believed that the GWAS data with a high quality could leads to the results with a high accuracy.

414 Note that our European GWAS data had been QCed before, the variants with MAF $<0.5 \%$ had

415 been removed, which meant that the imputation performance on rare variants of European

416 population were not available. However, we included all variants in Han Chinese GWAS data,

417 even for singletons. The common variants can be accurately imputed by any existing big

418 reference panel, such as the $1000 \mathrm{G}$ and the HRC panel. We used the rare variants set in Han

419 Chinese to study the genotyping imputation accuracy changes patterns with different composition

420 of reference panels.

421 All the customized reference panels in this study were based on the $1000 \mathrm{G}$ Phase 3 , there were

422 two popular versions for the $1000 \mathrm{G}$ panel, one included singletons and another did not. We found

423 that the Sanger Imputation Server used singleton-included version and the Michigan Imputation

424 Server used non-singleton version. We investigated the imputation performance of these two

425 versions of panel on Han Chinese data in local and found that the difference between them were

426 negligible (Supplementary Figure 5 and Supplementary Table 3). After all, we decided to use the

427 non-singleton version and were consistent with Michigan server since the imputation tool that we

428 employed were both Minimac3. The customized reference panels fell into three categories: 1)

429 haplotype size changed and the population diversity ratio was fixed. 2) Population diversity

430 degree changed and the haplotype size was fixed. 3) Both of them changed with a fixed step-size, 
431 note that the increased diversity ratio became more and more small because the panel size got

432 more and more large. In the design of our second category panels, we made a simple cluster

433 analysis by the longitude and latitude of populations, the result showed that almost all populations

434 obviously followed by the $1000 \mathrm{G}$ groups (EAS, EUR, AFR, AMR and SAS), except the 'ACB'

435 population (Supplementary Figure 8). The 'ACB' was classified into the AFR group in the 1000G,

436 but it was far more close to the AMR group geographically, so we reclassified this population

437 into the AMR group. But in reality, it will not make a nontrivial difference in our study, because

438 our GWAS set were Han Chinese and European population, the 'ACB' was always diverse to our

439 study sets. Beside the haplotypes size and diversity of reference panel, the sequencing coverage

440 would also affect the imputation accuracy. The high-depth sequencing could result the more

441 accurate genotypes in the reference panel, which in turn improve the accuracy of the inferred

442 haplotypes[1]. We did not systematically evaluate the influence of sequencing coverage since the

443 rest of effective variables could not be completely controlled by the update public reference

444 panels.

445 There was a phenomenon that crossed all the imputation results, which is that the imputation

446 performance for the European population was always better than for Han Chinese by their best-

447 panels. Taking the first category panels (described in the previous paragraph, imputation accuracy

448 vs. haplotype size) as an example, the results showed that the best-panel (the CHB panel) for the

449 Han Chinese population got a 0.874 average accuracy while the European got a 0.947 average

450 accuracy by its best-panel (the CEU panel). The first reason that came to mind might cause this

451 gap was that the European GWAS data not included variants with MAF $<0.5 \%$, but the Han

452 Chinese did, even if the variants between them were shared. We then removed all variants with 
453 MAF less than $0.5 \%$ that in Han Chinese data for both sets, and found the average imputation

454 accuracy raised to 0.929 and 0.948 for the Han Chinese and European respectively, the gap

455 narrowed but still existed. We could know it from the statistic of variants of different MAF bins

456 as well (Figure $2 \mathrm{c}$ and $2 \mathrm{~d}$ ). Another reason might cause the gap was the microarray chip, the two

457 GWAS sets used the Illumina 610k BeadChips which was designed for the European population

458 at the beginning.

459 Another discrepancy of imputation results between the Han Chinese and European population

460 was that, despite the introduced diversity, the accuracy of imputed common variants of European

461 population could always benefit from the expanding haplotypes size of reference panel, while the

462 corresponding accuracy of the Han Chinese population could benefit only when the diversity of

463 reference panel remained a small ratio (Figure 5). This result suggested that, in a sense, the

464 genome of the European may have a higher acceptability than Chinese genome which meant it

465 was more diverse. In the course of evolution, the view of intermarriage of Chinese was more

466 conservative than European[38, 39]. And an open intermarriage view may result in the genome

467 became more and more diverse across generations.

468 In the last decade, many cohort studies and WGS projects have been conducted, and several

469 genome reference panels were produced. However, most of the these cohorts and reference panels

470 were focused on the European and African American populations, such as the Wellcome Trust

471 Case Control Consortium (WTCCC)[40], UK10K[24], HRC[26] and TOPMed program. Few

472 WGS projects were conducted in Chinese population. The HapMap3 only included 137 native

473 Chinese individuals[20], the $1000 \mathrm{G}$ project phase 3 included 301 Chinese individuals, and only 
474208 were Han Chinese[23]. In 2017, 90 unrelated individuals of Chinese ancestry were sequenced

475 at a high depth $(\sim 80 X)[41]$ by the Beijing Genomics Institute (BGI-Shenzhen). However, the

476 sample size of these WGS projects was small. Although the CONVERGE project sequenced

47711,670 female Han Chinese and provided the largest whole genome sequencing resource of

478 Chinese[27], it was only able to call $\sim 22$ million high quality variants and the sequencing

479 coverage of CONVERGE was low (1.7X). At present, we are engaging in a Chinese cohort and

480 have collected $10 \mathrm{~K}$ samples across 29 provincial regions of China, the sequencing of $\sim 4000$

481 samples at $\sim 17 \mathrm{X}$ average coverage is ongoing. We hope to generate a high quality and decent

482 population-specific reference panel for public use for the largest ethnic group in the world.

In summary, we systematically investigated the relationship between genotype imputation

484 accuracy of rare variants and the composition of reference panel, and proposed an optimum

485 constituent ratio for the reference panel for the Han Chinese imputation. We found the different

486 patterns of imputation accuracy variation between the European and Han Chinese. This point enlightened us that we should use more special panels when impute the Chinese genome, and this could be generalized to the other populations with conservative genome.

\section{Acknowledgments}

492 This study was supported by the Zhejiang Provincial Natural Science Foundation for

493 Distinguished Young Scholars of China (LR17H070001) and by the National Natural Science

494 Foundation of China (81871831). The funding agencies had no role in the study design, data 
bioRxiv preprint doi: https://doi.org/10.1101/642546; this version posted May 19, 2019. The copyright holder for this preprint (which was not certified by peer review) is the author/funder. All rights reserved. No reuse allowed without permission.

496

reviewers for their thorough and helpful review of this manuscript. 


\section{References:}

1. Das S, Abecasis GR, Browning BL: Genotype Imputation from Large Reference Panels. Annu Rev Genomics Hum Genet 2018, 19:73-96.

2. Mahajan A, Taliun D, Thurner Met al: Fine-mapping type 2 diabetes loci to single-variant resolution using high-density imputation and islet-specific epigenome maps. Nat Genet 2018, 50(11):1505-1513.

3. Zheng HF, Forgetta V, Hsu YH et al: Whole-genome sequencing identifies EN1 as a determinant of bone density and fracture. Nature 2015, 526(7571):112-117.

4. Estrada K, Styrkarsdottir U, Evangelou E et al: Genome-wide meta-analysis identifies 56 bone mineral density loci and reveals 14 loci associated with risk of fracture. Nat Genet 2012, 44(5):491-501.

5. Willer CJ, Sanna S, Jackson AU et al: Newly identified loci that influence lipid concentrations and risk of coronary artery disease. Nat Genet 2008, 40(2):161-169.

6. Anderson CA, Pettersson FH, Barrett JC et al: Evaluating the effects of imputation on the power, coverage, and cost efficiency of genome-wide SNP platforms. Am J Hum Genet 2008, 83(1):112-119.

7. Lin Y, Liu L, Yang S et al: Genotype imputation for Han Chinese population using Haplotype Reference Consortium as reference. Hum Genet 2018, 137(6-7):431-436.

8. Vergara C, Parker MM, Franco L et al: Genotype imputation performance of three reference panels using African ancestry individuals. Hum Genet 2018, 137(4):281-292.

9. Zheng HF, Ladouceur M, Greenwood CM et al: Effect of genome-wide genotyping and reference panels on rare variants imputation. J Genet Genomics 2012, 39(10):545-550.

10. Gibson G: Rare and common variants: twenty arguments. Nat Rev Genet 2012, 13(2):135-145.

11. Barbujani G, Colonna V: Human genome diversity: frequently asked questions. Trends Genet 2010, 26(7):285-295.

12. International Multiple Sclerosis Genetics Consortium. Electronic address ccye, International Multiple Sclerosis Genetics C: Low-Frequency and Rare-Coding Variation Contributes to Multiple Sclerosis Risk. Cell 2018, 175(6):1679-1687 e1677.

13. Tin A, Li Y, Brody JA et al: Large-scale whole-exome sequencing association studies identify rare functional variants influencing serum urate levels. Nat Commun 2018, 9(1):4228.

14. Gazal S, Loh PR, Finucane HK et al: Functional architecture of low-frequency variants 
studies by imputation of genotypes. Nat Genet 2007, 39(7):906-913.

19. Huang L, Li Y, Singleton $A B$ et al: Genotype-imputation accuracy across worldwide human populations. Am J Hum Genet 2009, 84(2):235-250.

20. International HapMap C, Altshuler DM, Gibbs RA et al: Integrating common and rare genetic variation in diverse human populations. Nature 2010, 467(7311):52-58.

21. Huang J, Howie B, McCarthy S et al: Improved imputation of low-frequency and rare variants using the UK10K haplotype reference panel. Nat Commun 2015, 6:8111.

22. Deelen $\mathrm{P}$, Menelaou A, van Leeuwen EM et al: Improved imputation quality of low-frequency and rare variants in European samples using the 'Genome of The Netherlands'. Eur J Hum Genet 2014, 22(11):1321-1326.

23. Genomes Project C, Auton A, Brooks LD et al: A global reference for human genetic variation. Nature 2015, 526(7571):68-74.

24. Consortium UK, Walter K, Min JL et al: The UK10K project identifies rare variants in health and disease. Nature 2015, 526(7571):82-90.

25. Boomsma DI, Wijmenga C, Slagboom EP et al: The Genome of the Netherlands: design, and project goals. Eur J Hum Genet 2014, 22(2):221-227.

26. McCarthy S, Das S, Kretzschmar W et al: A reference panel of 64,976 haplotypes for genotype imputation. Nat Genet 2016, 48(10):1279-1283.

27. Cai N, Bigdeli TB, Kretzschmar WW et al: 11,670 whole-genome sequences representative of the Han Chinese population from the CONVERGE project. Sci Data 2017, 4:170011.

28. Nelson SC, Stilp AM, Papanicolaou GJ et al: Improved imputation accuracy in Hispanic/Latino populations with larger and more diverse reference panels: applications in the Hispanic Community Health Study/Study of Latinos (HCHS/SOL). Hum Mol Genet 2016, 25(15):32453254 .

29. Lert-Itthiporn W, Suktitipat B, Grove H et al: Validation of genotype imputation in Southeast Asian populations and the effect of single nucleotide polymorphism annotation on imputation outcome. BMC Med Genet 2018, 19(1):23.

30. Jostins L, Morley KI, Barrett JC: Imputation of low-frequency variants using the HapMap3 benefits from large, diverse reference sets. Eur J Hum Genet 2011, 19(6):662-666.

31. Han JW, Zheng HF, Cui Y et al: Genome-wide association study in a Chinese Han population identifies nine new susceptibility loci for systemic lupus erythematosus. Nat Genet 2009, 
2019, 2:9.

37. Shendure J, Balasubramanian S, Church GM et al: DNA sequencing at 40: past, present and future. Nature 2017, 550(7676):345-353.

38. Jian Z: The recent trend of ethnic intermarriage in China: an analysis based on the census data. The Journal of Chinese Sociology 2017, 4(1):11.

39. Alba RD: Intermarriage and ethnicity among European Americans. Contemporary Jewry 1991, 12(1):3-19.

40. Wellcome Trust Case Control C, Craddock N, Hurles ME et al: Genome-wide association study of CNVs in 16,000 cases of eight common diseases and 3,000 shared controls. Nature 2010, 464(7289):713-720.

41. Lan $\mathrm{T}$, Lin $\mathrm{H}$, Zhu W et al: Deep whole-genome sequencing of $90 \mathrm{Han}$ Chinese genomes. Gigascience 2017, 6(9):1-7. 


\section{Figure legends}

\section{Figure 1. Research design.}

(a) The design of imputation accuracy vs. reference panel size. 24 worldwide population of the 1000G

Phase3 were selected (sample size > 85), such CHB, CEU and GIH. For each population, the haplotypes were extracted and customized as reference panels with 4 sizes gradients $(50,90,130,170$ haplotypes). Totally, 96 reference panels (24 times 4) were formed, and then the imputation were performed for the Han Chinese and European chip data in local server. (b) The design of imputation accuracy vs. reference panel diversity. In this part, the size of reference panels was fixed to 640 haplotypes. 25 populations of the $1000 \mathrm{G}$ Phase 3 were selected and categorized into 5 groups (EAS, EUR, SAS, AFR and AMR, see Methods), each group included 5 populations and each population contained 64 samples. The 5 groups of population that we considered corresponded to a set of vectors ( $i_{1}$ to $i_{5}$ ), and solved the function of $i_{1}+i_{2}+i_{3}+i_{4}+i_{5}=5$. We got 126 positive integer solutions in total, which represented 126 combinations. Finally, 126 reference panels were formed and the imputations were performed for the Han Chinese and European chip data in local server. (c) The design of imputation accuracy vs. reference panel size \& diversity. 12 populations were selected. 9 of them were diverse to Han Chinese and European population respectively. Then, the CHB and CEU were used as basic panel, the diverse samples were recursively added to them, 9 samples per time and 85 times in total. Finally, two reference panels set were formed, and each group included 86 reference panels $(1+85)$. The imputations were then performed for the Han Chinese and European chip data in local server. (d) Imputation for the Han Chinese and European population using 1000G, HRC and CONVERGE reference panels.

Figure 2. Imputation accuracy for the reference panels with four haplotype size gradients.

Overall imputation accuracy for (a) the Han Chinese and (b) European using 24 worldwide populations of the $1000 \mathrm{G}$ Phase 3 as reference panels. For each reference panel, the average $\mathrm{EmpR}^{2}$ (measuring the imputation accuracy) was plotted with four haplotype sizes (50, 90, 130 and 170). (c) Imputation accuracy for the Han Chinese in 5 different MAF bins using CHB (Han Chinese ancestry) population as the reference panel. (d) Imputation accuracy for the European using CEU (European ancestry) 
(upper) was for the Han Chinese imputation and the asterisk marked group (lower) was for the European imputation.

Figure 4. Imputation accuracy for the reference panels with different diversity degrees.

(a) The boxplot of the EmpR ${ }^{2}$ for Han Chinese using the reference panels with different proportion of EAS populations. Since our study data was Han Chinese population, we used the proportions of nonEAS samples of $1000 \mathrm{G}$ ( $\sim 0$ to $100 \%)$ represented the diversity degrees $\left(\right.$ level $_{0}$ to level $\left._{5}\right)$ to the GWAS data. All variants were divided into $5 \mathrm{MAF}$ bins. The outliers (mean $\mathrm{EmpR}^{2}$ more than $\mathrm{Q}_{3}+1.5 * \mathrm{IQR}$ or less than $\left.\mathrm{Q}_{1}-1.5 * \mathrm{IQR}, \mathrm{IQR}=\mathrm{Q}_{3}-\mathrm{Q}_{1}\right)$ were not plotted. This plot was based on the 126 reference panels,

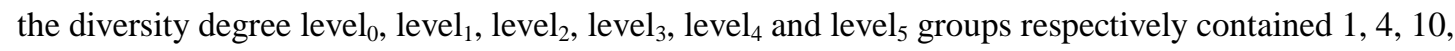
20, 35 and 56 reference panels. (b) The boxplot of the EmpR ${ }^{2}$ for European using the reference panels with different proportion of non-EUR populations. Similar to (a), but only three MAF bins of EmpR ${ }^{2}$ of variants were plotted since the variants with $\mathrm{MAF}<0.5 \%$ were not available (see Methods).

Figure 5. Imputation accuracy for the reference panels with population diversity and sample size constantly increased.

(a) Overall imputation accuracy trends for Han Chinese and European populations. For Han Chinese, the basic panel (0-step of additions) was CHB (0\% diversity, 103 samples with Han Chinese ancestry), the final reference panel was 85 -step of additions panel ( $88 \%$ diversity, 868 samples). For the European, the basic panel was CEU (0\% diversity, 99 samples with European ancestry). The final reference panel was 85-step of additions panel (88\% diversity, 864 samples). (b) Imputation accuracy trend for Han Chinese in 5 MAF bins using the reference panels with population diversity and sample size constantly increased. (c) Imputation accuracy trend for European, and only three MAF bins of EmpR ${ }^{2}$ of variants were plotted since the variants with $\mathrm{MAF}<0.5 \%$ were not available (see Methods).

Figure 6. Number of imputed variants for the 1000G, HRC and CONVERGE reference panels. 
(a) Imputation accuracy for the Han Chinese using four different reference panels. Only the mean $\mathrm{EmpR}^{2}$ of low-frequency and rare variants were plotted, and the variants were divided into 4 MAF bins.

The panel of "CHB + diversity (21\%)" was refer to CHB21D panel, which consisted of CHB population and $21 \%$ extra diverse samples (i.e. the 3-step panel for Han Chinese in last section). (b) Imputation accuracy for the European using three reference panels, and only three MAF bins of EmpR ${ }^{2}$ of variants were plotted since the variants with $\mathrm{MAF}<0.5 \%$ were not available (see Methods) 
the Han Chinese and European populations

\begin{tabular}{|c|c|c|c|c|c|}
\hline \multirow{2}{*}{$\mathrm{N}$} & \multirow{2}{*}{$\begin{array}{l}\text { Well-imputed variants number } \\
\text { (proportion) }\end{array}$} & \multicolumn{2}{|c|}{ Shared variants } & \multicolumn{2}{|c|}{ *Genotyped variants } \\
\hline & & $\mathrm{N}$ & Mean $\mathrm{R}^{2}(\mathrm{SD})$ & $\mathrm{N}$ & Mean $\mathrm{EmpR}^{2}$ (SD) \\
\hline $39,127,690$ & $6,228,449$ (15.9\%) & $10,302,818$ & $0.72(0.19)$ & 515,754 & $0.89(0.17)$ \\
\hline $47,109,465$ & $7,168,371(15.2 \%)$ & $10,302,818$ & $0.68(0.22)$ & 516,408 & $0.91(0.14)$ \\
\hline $47,109,431$ & $6,275,000(13.3 \%)$ & - & - & 516,408 & $0.91(0.16)$ \\
\hline $24,114,249$ & $5,626,185(23.3 \%)$ & $10,302,818$ & $0.58(0.20)$ & 511,715 & $0.92(0.11)$ \\
\hline $39,127,690$ & $12,871,067$ (32.9\%) & $10,302,818$ & $0.73(0.12)$ & 515,754 & $0.98(0.04)$ \\
\hline $47,109,465$ & $9,422,724(20.0 \%)$ & $10,302,818$ & $0.69(0.18)$ & 516,408 & $0.96(0.07)$ \\
\hline $24,114,249$ & $4,539,069$ (18.8\%) & $10,302,818$ & $0.51(0.18)$ & 511,715 & $0.82(0.21)$ \\
\hline
\end{tabular}

imputation were performed in local server, and the HRC-based imputation was performed in Michigan Imputation Server. The 1000G Phase 3 reference panel we used was the igan imputation server, and the both servers employed Minimac3 as the impute engine. $\mathrm{N}$ means the imputed sites number, and we defined the imputed variants with $\mathrm{R}^{2}$ more

Quad (610K) BeadChip, the imputation accuracy was measured by EmpR² (Empirical-R2, see methods). 


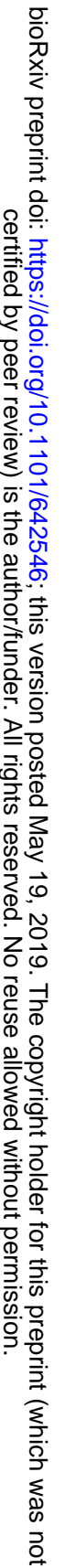




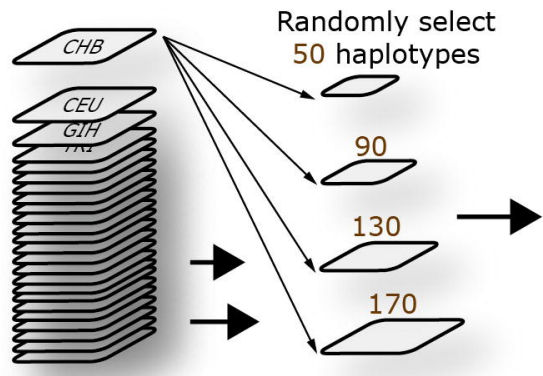

$24 \times 4$ reference panels

with 4 size gradients

Han Chinese data

\section{b}

$$
\begin{aligned}
& \text { EAS } \\
& \text { EUR } \\
& \text { SAS } \\
& \text { AFR } \\
& \text { AMR } \\
& \text { c }
\end{aligned}
$$

c

25 populations from the $1000 \mathrm{G}$

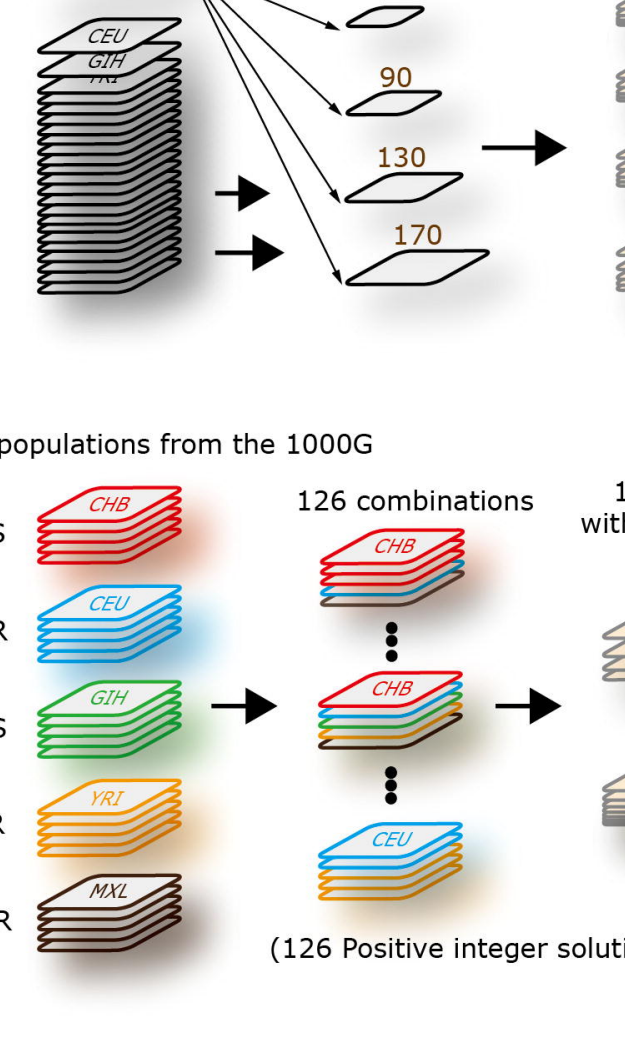
$(1+85) \times 2$ reference panels with raised size and diversity

6 reference panels

Han Chinese data 9 populat
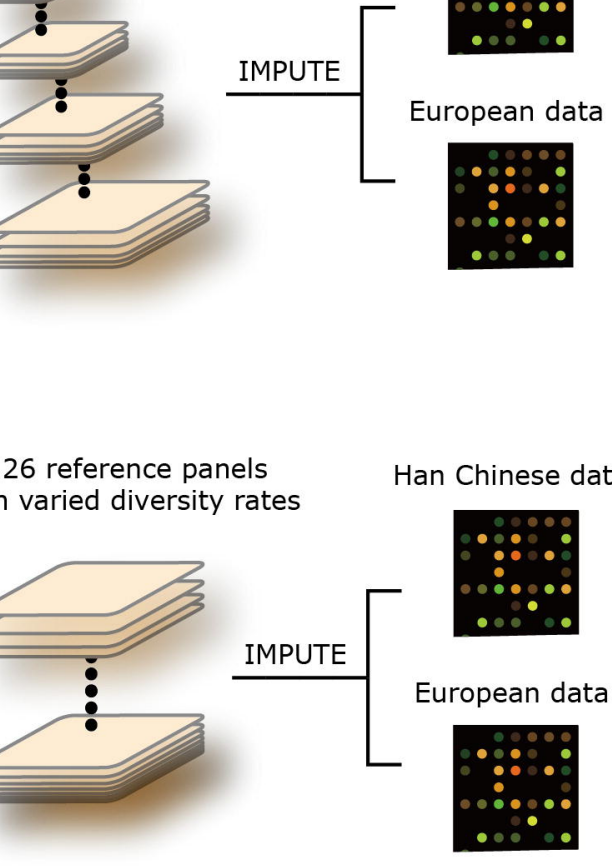
$\mathbf{a}$

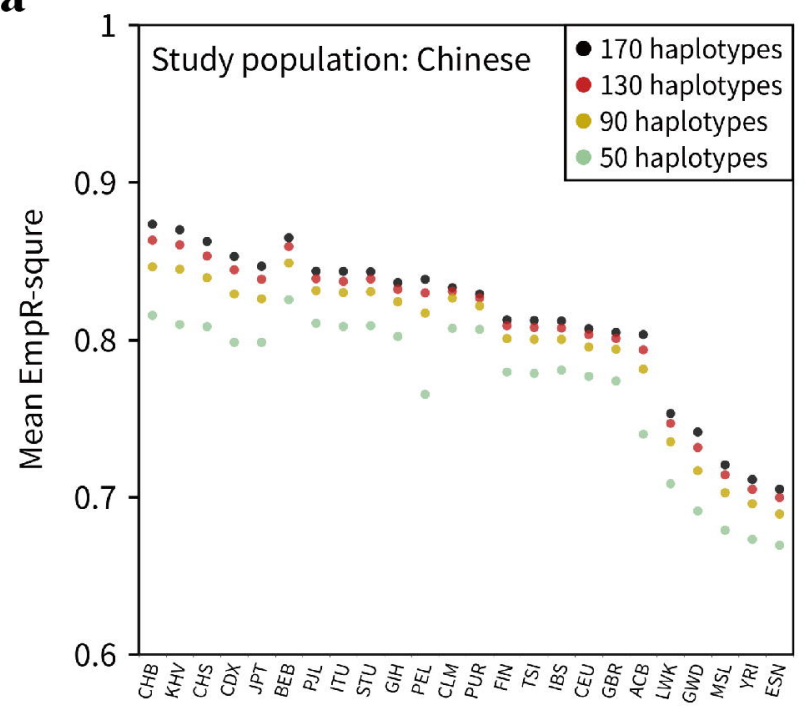

Population-specific panels of 1000GP

c

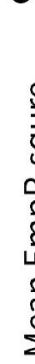

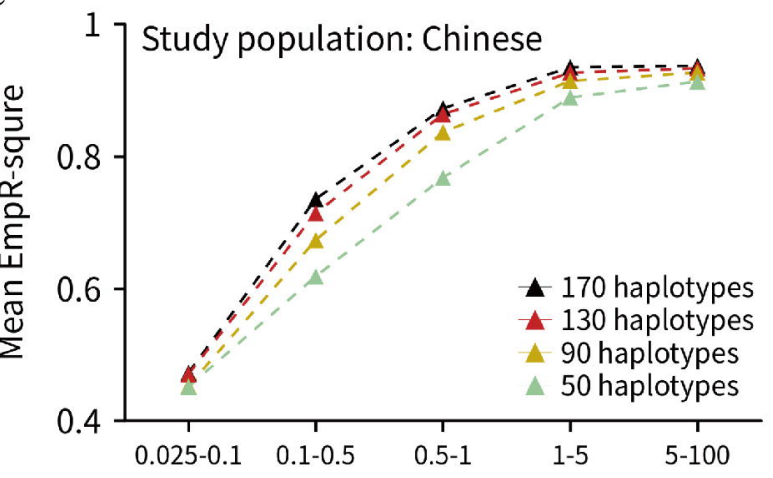

Minor allele frequency (\%)

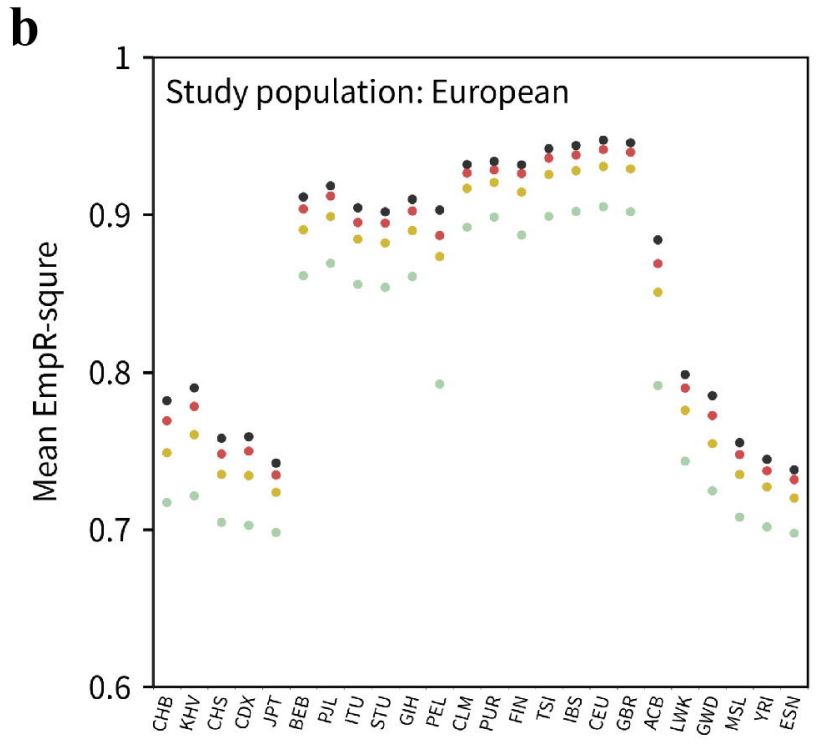

Population-specific panels of 1000GP
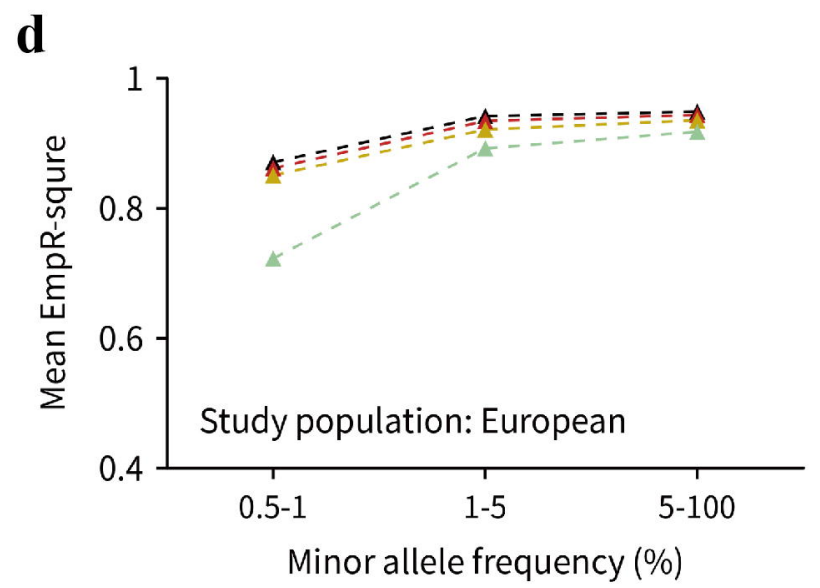
a

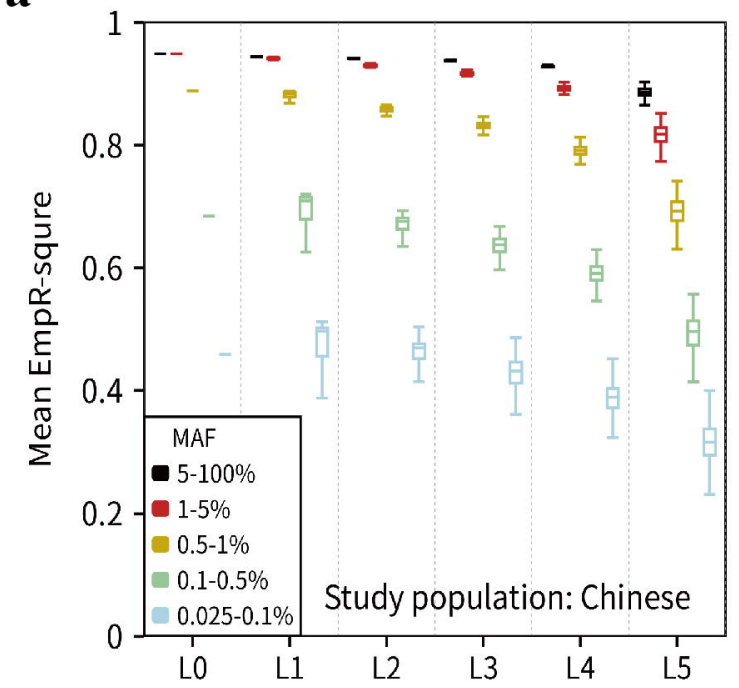

Reference panel diversity degree level (L) b

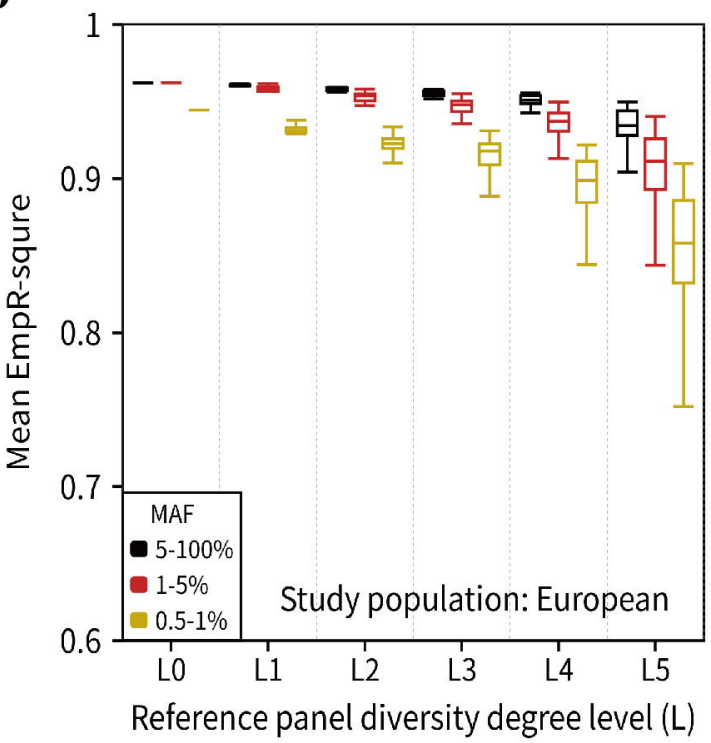



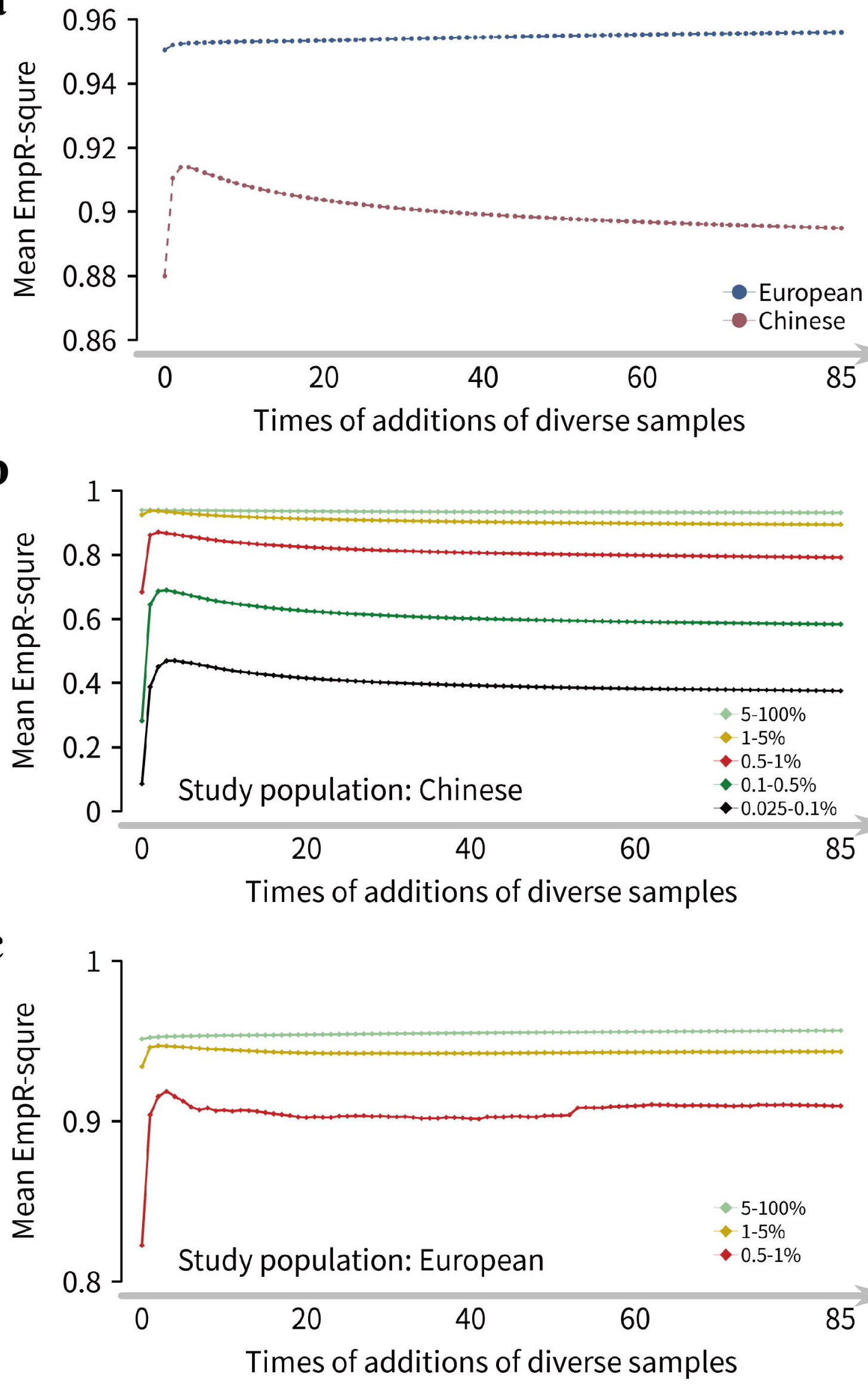


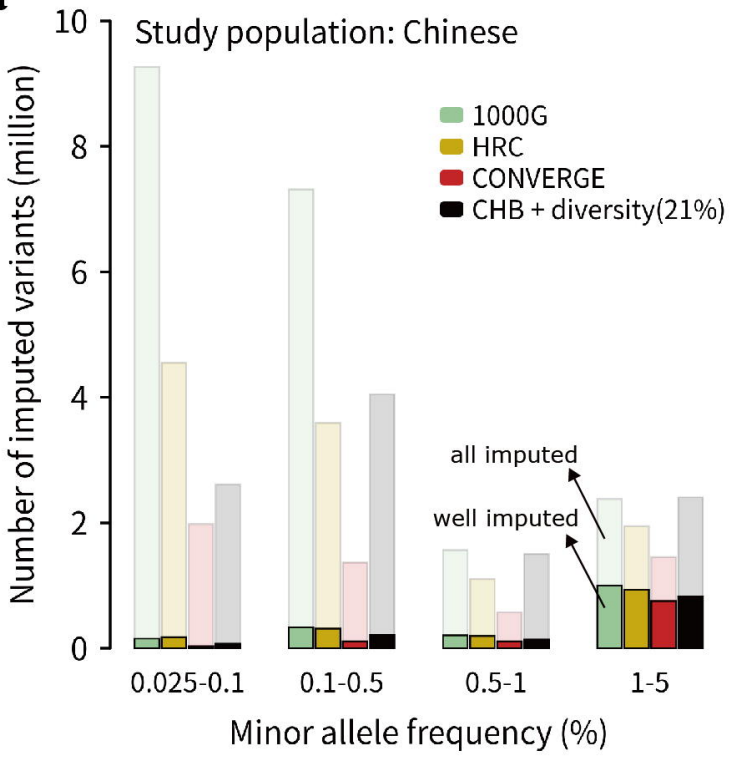

b

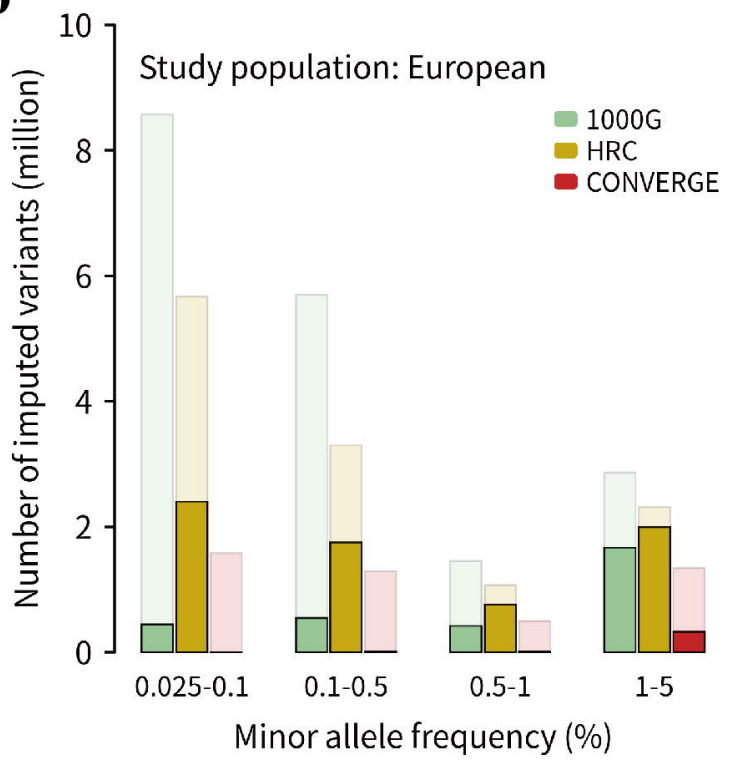


